\title{
Volumetric plasmonic resonator architecture for thin-film solar cells
}

Mustafa Akin Sefunc, Ali Kemal Okyay, and Hilmi Volkan Demir

Citation: Appl. Phys. Lett. 98, 093117 (2011);

View online: https://doi.org/10.1063/1.3560446

View Table of Contents: http://aip.scitation.org/toc/apl/98/9

Published by the American Institute of Physics

\section{Articles you may be interested in}

Tunable light trapping for solar cells using localized surface plasmons Journal of Applied Physics 105, 114310 (2009); 10.1063/1.3140609

Design principles for particle plasmon enhanced solar cells

Applied Physics Letters 93, 191113 (2008); 10.1063/1.3021072

Surface plasmon enhanced silicon solar cells

Journal of Applied Physics 101, 093105 (2007); 10.1063/1.2734885

Improved performance of amorphous silicon solar cells via scattering from surface plasmon polaritons in nearby metallic nanoparticles

Applied Physics Letters 89, 093103 (2006); 10.1063/1.2336629

Plasmonic nanoparticle enhanced light absorption in GaAs solar cells

Applied Physics Letters 93, 121904 (2008); 10.1063/1.2988288

A 2-terminal perovskite/silicon multijunction solar cell enabled by a silicon tunnel junction Applied Physics Letters 106, 121105 (2015); 10.1063/1.4914179

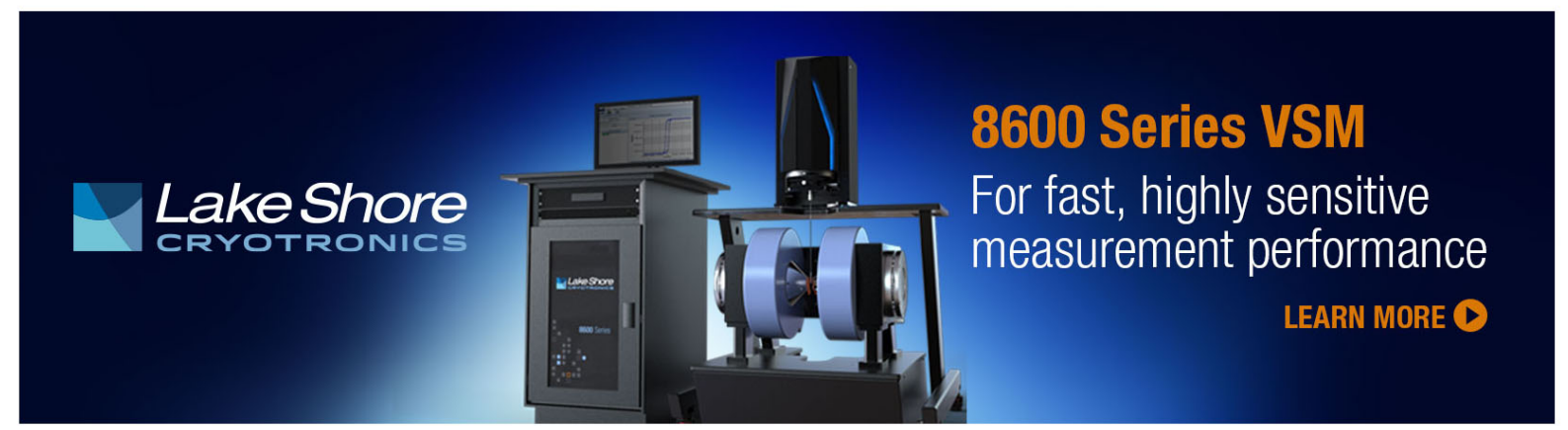




\title{
Volumetric plasmonic resonator architecture for thin-film solar cells
}

\author{
Mustafa Akin Sefunc, ${ }^{\text {a) }}$ Ali Kemal Okyay, and Hilmi Volkan Demir ${ }^{\text {b) }}$ \\ Department of Electrical and Electronics Engineering, Department of Physics, UNAM-Institute of Materials \\ Science and Nanotechnology, Bilkent University, Ankara 06800, Turkey and School of Electrical and \\ Electronic Engineering, School of Physical and Mathematical Sciences, Nanyang Technological University, \\ Nanyang Avenue, Singapore 639798
}

(Received 24 December 2010; accepted 21 January 2011; published online 4 March 2011)

\begin{abstract}
We propose and demonstrate a design concept of volumetric plasmonic resonators that relies on the idea of incorporating coupled layers of plasmonic structures embedded into a solar cell in enhanced optical absorption for surface-normal and off-axis angle configurations, beyond the enhancement limit of individual plasmonic layers. For a proof-of-concept demonstration in a thin-film organic solar cell that uses absorbing materials of copper phthalocyanine/perylene tetracarboxylic bisbenzimidazole, we couple two silver grating layers such that the field localization is further extended within the volume of active layers. Our computational results show a maximum optical absorption enhancement level of $\sim 67 \%$ under air mass 1.5 global illumination considering both polarizations. () 2011 American Institute of Physics. [doi:10.1063/1.3560446]
\end{abstract}

To combat environmental concerns ${ }^{1,2}$ escalating with increasing carbon footprint, along with the energy problem of limited resources, there has been a growing interest in decreasing the cost and/or increasing the efficiency of clean renewable energy sources including those of photovoltaic approaches for conversion of sunlight into electricity. Presently, although photovoltaics is considered as a potential candidate in diversification of energy sources, the cost of photovoltaic systems remains yet to be reduced by several factors to compete with fossil fuel based energy production. To this end next-generation solar cells are being investigated to feature very thin layers of active (absorbing) materials in the order of tens of nanometers. ${ }^{3,7}$ For example, such one promising organic solar cell is based on the use of absorbing materials of copper phthalocyanine $(\mathrm{CuPc})$ and perylene tetracarboxylic bisbenzimidazole (PTCBl), previously proposed and demonstrated by Forrest and co-workers. ${ }^{4}$ However, such ultrathin absorbing layers suffer from undesirably low optical absorption of incident photons.

Recently, efforts on increasing light trapping in the active photovoltaic materials have been demonstrated via using surface plasmon excitations. ${ }^{3-17}$ These prior studies used either randomly distributed metal nanoparticles ${ }^{3,6,7}$ or nanopatterned metal layers. ${ }^{3,8-14}$ Although such metal nanoparticles can be conveniently blended across the active layers, they suffer from random distribution in the active material and limited spatial control. On the other hand, the metal layers can be nanostructured with precision. But these use only a single nanopatterned layer of plasmonic structures, which limits the full utilization of the volume of the active material. All of these previous designs were based on placing the metal layer either only on the top $p^{3,7-10,15}$ or only at the bottom ${ }^{3,11-14}$ of the active layers for exciting their plasmon modes. The resulted absorption enhancement levels reported for such single layers have typically been limited within the range of $\sim 18 \%-50 \%$, when considering both transverse-magnetic (TM) and transverse-electric

(TE)

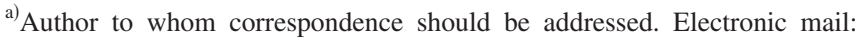
sefunc@ee.bilkent.edu.tr.

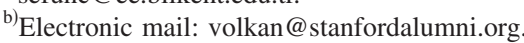

polarizations. ${ }^{8,9,12,13}$ Therefore, there is a need for further extending field localization across the volume of the active materials to contribute to increasing field localization and enhancing photon absorption efficiency in thin-film solar cells, beyond the improvement levels obtained using individual plasmonic layers. This, however, requires innovative designs. In this letter, to address this need, we report a volumetric design that couples two (or more) layers of plasmonic structures embedded in thin-film solar cells (Fig. 1).
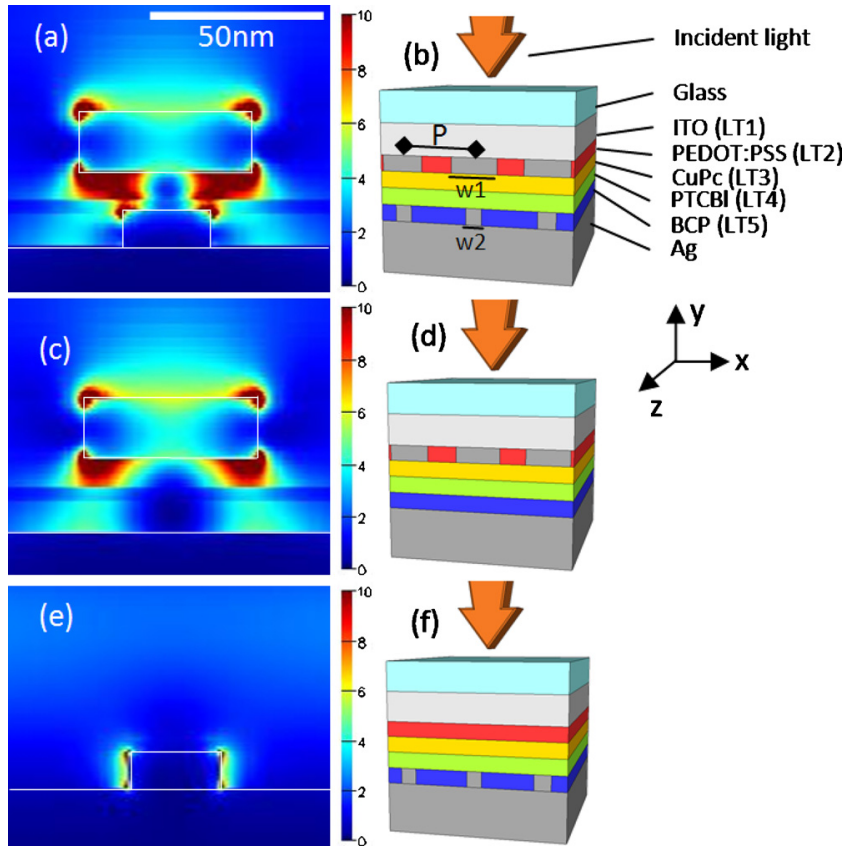

FIG. 1. (Color online) Computed electric field profiles (given as the squared field in $\mathrm{V}^{2} / \mathrm{m}^{2}$ ) for the thin-film device architectures: [(a) and (b)] Volumetric plasmonic design (with top and bottom gratings), [(c) and (d)] only top grating, and [(e) and (f)] only bottom grating. Here $P=200 \mathrm{~nm}$ (period of the gratings) (see Ref. 19), w1 $=50 \mathrm{~nm}$ (width of top metal grating), w2 $=30 \mathrm{~nm}$ (width of the bottom metal grating), and layer thicknesses of LT1 $=100 \mathrm{~nm}, \mathrm{LT} 2=20 \mathrm{~nm}, \mathrm{LT} 3=11 \mathrm{~nm}, \mathrm{LT} 4=4 \mathrm{~nm}$, and LT5=12 nm, under TM-polarized normal-incident illumination (illustrated with arrows) at $\lambda=510 \mathrm{~nm}$. A zoomed-in single unit cell is shown for easy visualization using the same color map in all of these field profiles. The scale bar is the same $(50 \mathrm{~nm})$ in all cases. 

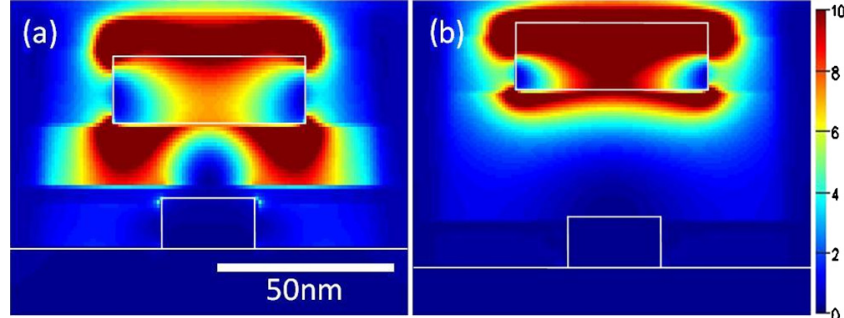

FIG. 2. (Color online) Computed normalized E-field distributions for different distances (a) 20 and (b) $50 \mathrm{~nm}$ between the top and bottom plasmonic resonators at $525 \mathrm{~nm}$ (enhanced online). [URL: http://dx.doi.org/10.1063/ 1.3560446.1]; [URL: http://dx.doi.org/10.1063/1.3560446.2]

For a proof-of-concept demonstration in thin-film organic photovoltaics, we computationally studied $\mathrm{CuPc}$ PTCBl based solar cells by embodying two silver gratings as shown in Figs. 1(a) and 1(b). In this thin-film organic solar cell of glass/indium thin oxide (ITO)/PEDOT:PSS/CuPc/ $\mathrm{PTCB} 1 /$ bathocuproine $(\mathrm{BCP}) / \mathrm{Ag}$, the backside silver contact (cathode layer) is covered by a transparent BCP layer that facilitates electron transport. The thin active layers include a $4 \mathrm{~nm}$ thick PTCBl electron acceptor layer and an $11 \mathrm{~nm}$ thick $\mathrm{CuPc}$ electron donor (hole acceptor) layer, deposited on BCP layer. The top transparent layer made of PEDOT:PSS [poly(3,4-ethylenedioxythiophene):poly(styrenesulfonate)] is the hole transport layer. The top transparent ITO layer provides electrical contact from the solar cell. In this volumetric architecture, the first grating is partially embedded in the PEDOT:PSS layer on the top while the second grating is placed on the bottom by extending the silver electrode into the $\mathrm{BCP}$ layer to match lining of the top grating.

In our analyses, we computed the field maps of metallic gratings integrated into $\mathrm{CuPc} / \mathrm{PTCBl}$ based solar cells for different cases [Figs. 1(a)-1(f)] and investigated the relative contribution to optical absorption enhancement for each case with respect to the bare device using two-dimensional finitedifference time-domain (FDTD) method (Lumerical Solu-
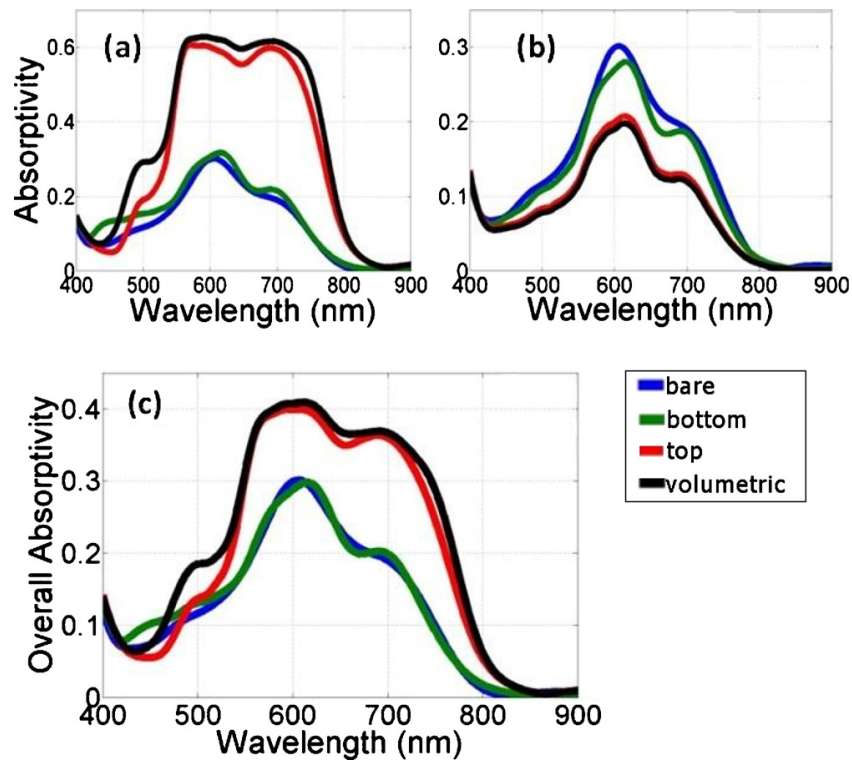

FIG. 3. (Color online) Computed absorption spectra of the active layers in the solar cell structures given in Fig. 1 under (a) TM- and (b) TE-polarized light, along with those of the bare solar cell without metallic grating structure for comparison. (c) Overall absorptivity $\left(\mathrm{A}_{\mathrm{TM}}+\mathrm{A}_{\mathrm{TE}}\right) / 2$ of the same device architectures (enhanced online). [URL: http://dx.doi.org/10.1063/ 1.3560446.3]; [URL: http://dx.doi.org/10.1063/1.3560446.4]

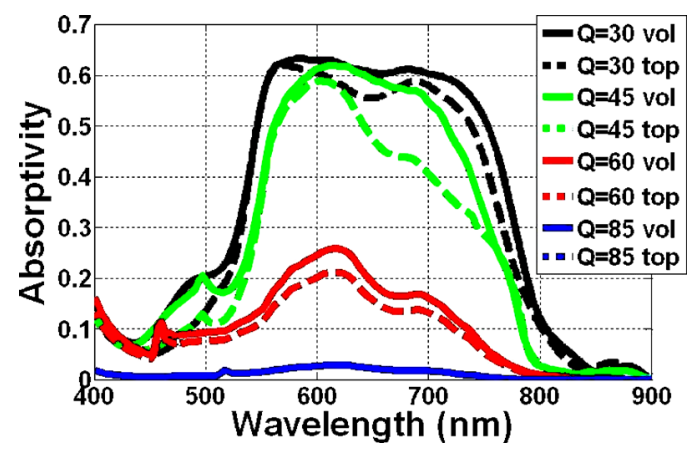

FIG. 4. (Color online) Effect of the incidence angle on total absorption enhancement under TM-polarized light. All device parameters are the same as those in Fig. 1.

tions Inc., Canada). In these numerical simulations, we calculated the frequency domain response by taking the Fourier transform of time domain representations. This computational approach allowed us to use experimental refractive index data to represent the device materials including $\mathrm{CuPc},{ }^{8}$ $\mathrm{PTCB} 1,{ }^{8} \mathrm{Ag},{ }^{16}$ and $\mathrm{BCP}^{17}$ in our structures. In all simulations, reflection from the air/glass interface was omitted. The simulation domain boundary conditions along $x$ axis were set to be periodic, while those along $y$ axis, to be perfectly matched.

We first studied the coupling of top and bottom gratings as a function of spacing between them. The vertical interaction between the top and bottom plasmonic resonators is dependent on the separating distance of these resonators. In Fig. 2, we considered two configurations for exemplifying the effect of thickness on the field distributions. In the Fig. 2(a) the separation between the top and bottom grating resonators is set to $20 \mathrm{~nm}$ while this is set to $50 \mathrm{~nm}$ in Fig. 2(b). As a result of transition from Fig. 2(a) to Fig. 2(b), we observed that the vertical coupling interaction is degraded with the increasing distance between resonators. In our optimized design, the separating thickness is set to $16 \mathrm{~nm}$. This allows us to take the advantage of the vertical interaction in the volumetric resonator in addition to individual plasmonic resonances of these metallic structures. This design enables strong nanoscale field confinement to increase optical absorption. ${ }^{18}$

We comparatively studied following three different cases: volumetric plasmonic resonator structure (consisting of coupled top and bottom silver gratings) [Figs. 1(a) and 1(b)], only top silver grating [Figs. 1(c) and 1(d)], and only bottom silver grating [Figs. 1(e) and 1(f)], with respect to the case of the starting solar cell with no metallic grating structures (dubbed "bare" device). The total absorptivity (Fig. 3) is calculated in the absorbing materials of CuPc and PTCB1 layers by

$$
A=\omega \times \operatorname{Im}(\varepsilon) \oint_{V}|E|^{2} d V^{\prime},
$$

where $E$ is the electric field, $V$ is the volume where the absorptivity is to be calculated, and is the dielectric constant of the material filling the volume. In the investigated cases, although the calculated absorbance is mostly dominated by the CuPc layer (because it is thicker than PTCBl layer), we included both the $\mathrm{CuPc}$ and PTCBl layers in our calculations. Here these device structures are illuminated by nor- 
mally incident planewave with TM-polarized illumination, i.e., $\mathrm{A}_{\mathrm{TM}}\left[\right.$ Fig. 3(a)] and TE-polarized illumination, i.e., $\mathrm{A}_{\mathrm{TE}}$ [Fig. 3(b)], which are used to compute the overall absorptivity, $\left(\mathrm{A}_{\mathrm{TM}}+\mathrm{A}_{\mathrm{TE}}\right) / 2$.

Computational results show that the electric field between the metallic gratings is locally amplified, and that the overall optical absorption is enhanced as a result of excitation of plasmonic modes in the top grating and volumetric design especially for the TM-polarized illumination [Fig. 3(a)]. This is attributed to broad excitation band of plasmonic modes between 480 and $850 \mathrm{~nm}$. However, under TEpolarized light, the absorption spectra of these active layers exhibit a reduced absorption level. Metallic gratings reflect part of the incident light, and also the thin active layers do not support any TE modes, explaining the suppression in their absorption spectra. Our proposed design based on using two plasmonic resonators introduces vertical coupling between them [Fig. 1(a)]. This is attributed to the interaction of localized plasmonic resonances excited around the top metallic structures and the surface plasmons associated with the bottom metallic grating. As a result, substantial electric field localization is observed in the absorbing materials, contributing to the enhancement of optical absorptivity. We compute the increase in absorption performance under AM1.5G (air mass 1.5 global) solar radiation (see Ref. 19) for the equation used in this computation). The numerical simulation results show that the volumetric design enhances the overall absorption $\left(\mathrm{A}_{\mathrm{TE}}+\mathrm{A}_{\mathrm{TM}}\right) / 2$ in this solar cell up to a maximum level of $\sim 67 \%$ under AM1.5G radiation, while a maximum possible enhancement level for the top grating layer alone is $\sim 58 \%$ and that for the bottom grating alone is $\sim 8 \%$. Here the maximum possible absorptivity is studied also considering the effect of grating periodicity (see Ref. 19) for the comparison of the top and volumetric resonator architectures). Although it is more difficult to fabricate such multilayer coupled plasmonic structures, the volumetric approach shows that it is in principle possible to exceed the enhancement levels of individual plasmonic players. However, this comes at the cost of increased fabrication complexity.

The volumetric design and the top grating exhibit improved enhancement levels compared to the bottom structure and the bare device for surface-normal configuration. In Fig. 4 , we investigate how much their performance is degraded as the angle of incidence is increased from surface normal toward off-axis angles for the volumetric design and the top grating. The optical absorption in the active layers decreases with the increasing angle due to the large reflection of the incoming light for both architectures. However, the drop is significantly faster in the case of the top grating alone, especially at long wavelengths. For example, at $45^{\circ}$, the top grating suffers noticeable reduction in the absorptivity while the volumetric design appears to be more tolerant to the angle change. But eventually the performance of the two structures converges at extremely shallow angles, like $85^{\circ}$ as shown. According to these simulations, the resulting absorption enhancement is relatively weakly dependent on the angle of incidence for small angles in the case of the volumetric design.

In conclusion, we proposed a volumetric design based on integrating two plasmonic resonators for enhancing the optical absorption in CuPc/PTCBl based solar cells, beyond the limit of single plasmonic resonator structures. In our computational analysis, we demonstrate that these vertically coupled top and bottom plasmonic layers enable a strongly localized electric field further extended across the volume of the absorbing layers. In addition to individual plasmonic resonances of these metallic structures, this vertical interaction in the volumetric resonator further contributes to the optical absorption enhancement in the active layer, reaching a maximum level of $\sim 67 \%$ under AM1.5G illumination considering both TM and TE polarizations while keeping the total device thickness fixed. This design strategy can potentially be extended to different types of organic and inorganic solar cells and three-dimensional structures.

This work is supported by NFR-RF, ESF-EURYI, EUFP7 Nanophotonics4Energy NoE, and TUBITAK EEEAG Grant nos. 107E088, 109E002, 109E004, 110E010, and 110E217. H.V.D. acknowledges support from TUBA-GEBIP.

${ }^{1}$ Intergovernmental Panel on Climate Change (IPCC), Climate Change 2007: The Physical Science Basis Fourth Assessment Report Summary for Policymaker, 2007.

${ }^{2}$ U.S. Environmental Protection Agency (EPA), Inventory of U.S. Greenhouse Gas Emissions and Sinks: 1990-2005, 2007.

${ }^{3}$ H. A. Atwater and A. Polman, Nature Mater. 9, 205 (2010).

${ }^{4}$ P. Peumans, S. Uchida, and S. R. Forrest, Nature (London) 425, 158 (2003).

${ }^{5}$ M. Agrawal and P. Peumans, Opt. Express 16, 5385 (2008).

${ }^{6}$ D. Duche, P. Torchio, L. Escoubas, F. Monestier, J. Simon, F. Flory, and G. Mathian, Sol. Energy Mater. Sol. Cells 93, 1377 (2009).

${ }^{7}$ S. H. Lim, W. Mar, P. Matheu, D. Derkacs, and E. T. Yu, J. Appl. Phys. 101, 104309 (2007).

${ }^{8}$ C. Min, J. Li, G. Veronis, J. Lee, S. Fan, and P. Peumans, Appl. Phys. Lett. 96, 133302 (2010).

${ }^{9}$ R. Pala, J. White, E. Barnard, J. Liu, and M. L. Brongersma, Adv. Mater. (Weinheim, Ger.) 21, 3504 (2009).

${ }^{10}$ F. J. Beck, S. Mokkapati, A. Polman, and K. R. Catchpole, Appl. Phys. Lett. 96, 033113 (2010).

${ }^{11}$ V. E. Ferry, L. A. Sweatlock, D. Pacifici, and H. A. Atwater, Nano Lett. 8, 4391 (2008).

${ }^{12}$ V. E. Ferry, M. A. Vershuuren, H. B. T. Li, R. E. I. Schropp, H. A. Atwater, and A. Polman, Appl. Phys. Lett. 95, 183503 (2009).

${ }^{13}$ W. Bai, Q. Gan, F. Bartoli, J. Zhang, L. Cai, Y. Huang, and G. Song, Opt. Lett. 34, 3725 (2009)

${ }^{14}$ W. E. I. Sha, W. C. H. Choy, and W. C. Chew, Opt. Express 18, 5993 (2010).

${ }^{15}$ S. Mokkapati, F. J. Beck, A. Polman, and K. R. Catchpole, Appl. Phys. Lett. 95, 053115 (2009).

${ }^{16}$ E. D. Palik, Handbook of Optical Constants of Solids (Academic, New York, 1985).

${ }^{17}$ Z. T. Liu, C. Y. Kwong, C. H. Cheung, A. B. Djurisic, Y. Chan, and P.C. Chui, Synth. Met. 150, 159 (2005).

${ }^{18}$ Z. Yu, A. Raman, and S. Fan, Proc. Natl. Acad. Sci. U.S.A. 107, 17491 (2010).

${ }^{19}$ See supplementary material at http://dx.doi.org/10.1063/1.3560446 for the effect of silver grating periodicity on the absorptivity for the top resonator and volumetric resonator architectures and for the equation used in the computation of the increased absorption under AM1.5G solar radiation. 